\title{
Comparison of Two Handheld Digital Dual Inclinometry Techniques in the Measurement of Lumbar Flexion Active Range of Motion
}

\author{
James P. Fletcher, James David Taylor, Chris A. Carroll, and M. Blake Richardson
}

\begin{abstract}
Context: An accurate assessment of lumbar spine active range of motion (AROM) is clinically important. Dual inclinometry is recommended as the optimal technique for measuring lumbar flexion AROM; however, the procedures differ in the literature. Objective: To compare 2 different handheld digital dual inclinometry (HDDI) techniques for evaluating lumbar flexion AROM. Design: The study was a repeated-measures design consisting of 2 trials. Setting: Laboratory. Participants: A sample of 69 adult volunteers (28 men and 41 women; mean age 23.8 [2.4] y) without pain or injury to their back, hips, or abdomen for at least 3 months participated in the study. Intervention: Using standardized methods, 1 trained tester performed 2 different HDDI measurements of standing lumbar flexion AROM on each subject. Each subject performed one repetition of AROM lumbar flexion per HDDI measurement. The HDDI measures differed in the process for placing the upper inclinometer, with one technique identifying the upper landmark by skilled palpation of the T12 spinous process and the other technique by measuring 15-cm cephalad to the S2 region landmark to approximate the location of the T12 spinous process. Main Outcome Measures: A dependent $t$ test, Pearson correlation coefficient $(r)$, the $95 \%$ limits of agreement, and Bland-Altman plots were used to examine agreement between the techniques. Results: Dependent $t$ testing showed no significant differences between the techniques (mean difference $\left.=1.2^{\circ}, P=.11\right)$. A strong correlation existed between the 2 HDDI techniques $(r=.80, P<.001)$. The Bland-Altman plot illustrated that 64 of the 69 data points were within the $95 \%$ limits of agreement for the 2 techniques. Conclusions: The findings suggest that HDDI measurements of lumbar flexion AROM are comparable when using either of the 2 HDDI techniques described. Clinicians can make an evidence-based choice for using either method of measuring lumbar flexion AROM.
\end{abstract}

Keywords: assessment, spine, measurement, inclinometer, Schober, T12

Lumbar active range of motion (AROM) is routinely assessed by clinicians when examining for impairments that relate to symptoms and physical dysfunction. Accurate, reliable, and easy to perform lumbar AROM measurements are essential for determining impairment severity, as well as to monitor a patient's response to interventions, given that increasing spinal mobility is often regarded as an important treatment goal. ${ }^{1}$

Handheld dual inclinometry (HDI) is recommended by the American Medical Association for the measurement of lumbar AROM and is well described in multiple sources. ${ }^{2,3}$ One typical HDI method uses one inclinometer placed at the 12th thoracic spinous process (T12) and a second placed on the sacrum at the second sacral vertebrae (S2), in line horizontally with the bilateral posterior superior iliac spines. The sacral inclinometer allows the measurement process to take into account only the joint motion superior to the sacrum, whereas the T12 inclinometer allows the measurement process to take into account only the joint motion inferior to T12. Thus, the measurement excludes both the thoracic and the pelvis/hip range of motion from the total forward bending motion, resulting in a reasonably isolated measure of lumbar AROM.

The techniques presented in the literature for identifying the location for placement of the upper inclinometer in adults (eg, T12 landmark) vary. Two common approaches for identifying the upper landmark are by skilled palpation of the T12 spinous process or by

The authors are with the Department of Physical Therapy, University of Central Arkansas, Conway, AR, USA. Fletcher (fletcher@uca.edu) is corresponding author. measuring $15-\mathrm{cm}$ cephalad to the $\mathrm{S} 2$ region landmark to approximate the location of the T12 spinous process. ${ }^{3-6}$ The $15-\mathrm{cm}$ approach appears to be popular due to the ease and efficiency of use, particularly in light of the possibility that an examiner's ability to identify and localize the T12 spinous process can be a source of error and measurement variance. ${ }^{4}$ The basis for the $15-\mathrm{cm}$ approach, which has been called the modified-modified Schober method, is described in the literature, which essentially asserts that $15 \mathrm{~cm}$ approximates the height of the lumbar spine in adults. ${ }^{5,6}$

No published evidence was found comparing the results of lumbar AROM inclinometry using the 2 common approaches for determining the location for placement of the upper inclinometer (eg, T12 landmark). The purpose of this study was to compare 2 different handheld digital dual inclinometry (HDDI) techniques for measuring lumbar flexion AROM.

\section{Methods}

A convenience sample of 69 healthy adult volunteers participated in the study. Approval of the study by the University of Central Arkansas institutional review board was obtained prior to the data collection, and an informed consent was signed by each subject before participating in the study. Individuals were excluded as subjects if they had (1) back, hip, or abdominal pain at the time of the data collection, (2) a history of back, hip, or abdominal pain within the last 3 months for which they sought treatment, (3) selfreported pregnancy beyond the first trimester, or (4) chronic dizziness. 
The examiner was a physical therapist with extensive training and experience in using the digital inclinometer and possessed the skills essential to standardizing the measurement process and minimizing error; such skills include identification of bony landmarks through palpation, performing stable and consistent placement of the instrument, and giving clear and accurate instruction to subjects regarding performance of movement. The device used to measure lumbar flexion AROM was an Acumar ${ }^{\mathrm{TM}}$ HDDI (Lafayette Instrument Co, Lafayette, IN). The device contains a microprocessor that calculates and displays the range of motion on a numerical liquid crystal display screen in $1^{\circ}$ increments with a reported measurement error of $\pm 1^{\circ}$. $^{7}$

Intrarater reliability of the measurement techniques was investigated using a subset of study participants $(n=16)$. The following procedures were used during the reliability study and, subsequently, during the data collection portion of the study. Each subject performed a warm-up to ensure familiarization and to prevent any unintended testing effects. The warm-up was explained and demonstrated, and then the subject was allowed to practice. Each subject performed 5 warm-up repetitions in a full range of thoracolumbar and pelvic-hip flexion in a standing posture (eg, forward bending), holding each repetition for 5 seconds. The subject then immediately transitioned into the data collection process where each subject underwent, in random order based on a card draw, 2 lumbar AROM HDDI measurements. All anatomical landmarks were palpated by the examiner, with the subject in upright standing, consistent with established techniques..$^{2-4,6,8}$ In one measurement, the landmarks were the palpated T12 spinous process and the palpated $\mathrm{S} 2$ region. In the other measurement, the landmarks were the palpated S2 region and $15-\mathrm{cm}$ cephalad to it. Each subject stood erect in a self-selected natural and comfortable position, with arms at their side and feet placed on footprint outlines marked on the floor (heels $15 \mathrm{~cm}$ apart from posterior medial borders). The 0 , or neutral, position was operationally defined as the subject's self-selected standing posture. The examiner then exposed the lumbosacral surface anatomy. To assure proper palpation and identification of landmarks, the male subjects were asked to remove their shirts, and the female subjects were given the option of raising their shirt high enough to expose the full lumbar spine or wearing a sports bra with no shirt. Prior to the first measurement, the inclinometers (Lafayette Instrument Co) were placed over the S2 region and the designated superior landmark (eg, T12 palpation vs $15-\mathrm{cm}$ approach) and zeroed with the spine in the selfselected neutral position. Landmarks were marked on the skin by a small dot made with a washable marker. The trial 1 data were collected for one of the 2 randomly ordered HDDI techniques by giving the following instructions: "bend forward as far as possible with your hands reaching toward your toes." The landmark markings were wiped off after each measurement with an alcohol pad. The subject then walked $20 \mathrm{ft}$ to have the body height and body weight measured. The subject was then immediately directed back for the trial 2 data collection (eg, the other randomly ordered HDDI technique) where landmarks were located and marked again. See Figure 1 for illustrations of the measurement techniques. Previous research indicates that the techniques used in this study for palpating and identifying the landmarks are reliable. ${ }^{8}$ For each measure, the examiner was blinded from the inclinometer's (Lafayette Instrument Co) digital readout, and an observer viewed the screen to ensure proper function and silently read and recorded all measurements to limit bias from the examiner.

Means and SDs were calculated for age, body mass, height, and body mass index to determine the participants' characteristics.
Normal distribution of data was examined using the Shapiro-Wilk test. Intrarater reliability of the 2 techniques was evaluated using the intraclass correlation coefficient (ICC) at an alpha level of .05. Differences between the 2 techniques were evaluated using a dependent $t$ test. Pearson's correlation coefficient $(r)$ was used to assess the relationship between the 2 techniques. A scatter plot was used to visually illustrate the correlation between both techniques. Bland-Altman plots with $95 \%$ limits of agreement (LOA) were used to illustrate visual representations of the measurement errors against true values by plotting the difference between the 2 measurements versus the mean of the 2 measurements. The $95 \%$ LOA were defined as 1.96 SDs above and below the mean of the difference scores. A cut-off score for lumbar flexion AROM was identified using the SD approach. The participants who scored $\geq 1$ SD below the mean were classified as having impaired lumbar flexion AROM. A receiver operating characteristic curve was then used to examine the validity of using the cut-off score to classify participants. For all statistical analyses, an alpha level of .05 was used. All statistical analyses were conducted using IBM SPSS for Windows (version 25.0; SPSS Inc, Chicago, IL).

\section{Results}

A convenience sample of 69 healthy adults (28 men and 41 women) participated in this study. Descriptive characteristics of the study participants are presented in Table 1. The Shapiro-Wilk test indicated a normal distribution of data. The ICC for the intrarater reliability of the T12 and $15-\mathrm{cm}$ techniques were .79 (95\% confidence interval, .42-.94) and .92 (95\% confidence interval, .73-.98), respectively, with a SE of $<4^{\circ}$ for each technique. The group means and SDs for the T12 and $15-\mathrm{cm}$ techniques were $43.3^{\circ}$ $\left(10.1^{\circ}\right)$ and $42.1^{\circ}\left(9.2^{\circ}\right)$, respectively. Dependent $t$ testing showed no differences between the 2 HDDI techniques $(t=-1.61, P=.11)$. The Pearson correlation coefficient indicated a strong correlation between the 2 techniques $(r=.80, P<.001)$, using the guideline by Portney and Watkins ${ }^{9}$ whereby values above 0.75 indicate a good to excellent association. A scatter plot of T12 method scores versus the $15-\mathrm{cm}$ method indicated a positive and linear relationship. The mean difference and 95\% LOA between the 2 techniques were -1.19 and 13.1 to 10.7. A Bland-Altman plot (Figure 2) illustrated that 64 of the 69 data points were within the $95 \%$ LOA for the T12 method versus the $15-\mathrm{cm}$ method. For both measurement techniques, $1 \mathrm{SD}$ below the mean was equal to $33^{\circ}$ of lumbar flexion AROM. Thus, $33^{\circ}$ of lumbar flexion AROM was identified as the cut-off score to classify the participants, meaning that participants with $33^{\circ}$ or less of lumbar flexion AROM were classified as having impaired lumbar flexion AROM. As the $15-\mathrm{cm}$ method is currently recommended in the literature, the $15-\mathrm{cm}$ method was used as the reference for classification. When compared with the $15-\mathrm{cm}$ method, the area under the curve for the T12 method was 0.91 $(P<.001)$, with a sensitivity of 0.69 and a specificity of 0.89 for classifying the participants as having impaired lumbar flexion AROM.

\section{Discussion}

No significant difference was found in lumbar flexion AROM measurements between the 2 HDDI techniques. Both techniques are potentially useful for clinical measurement. Localizing and approximating T12 via tape measure (eg, 15-cm technique) for upper inclinometer (Lafayette Instrument Co) placement is the 

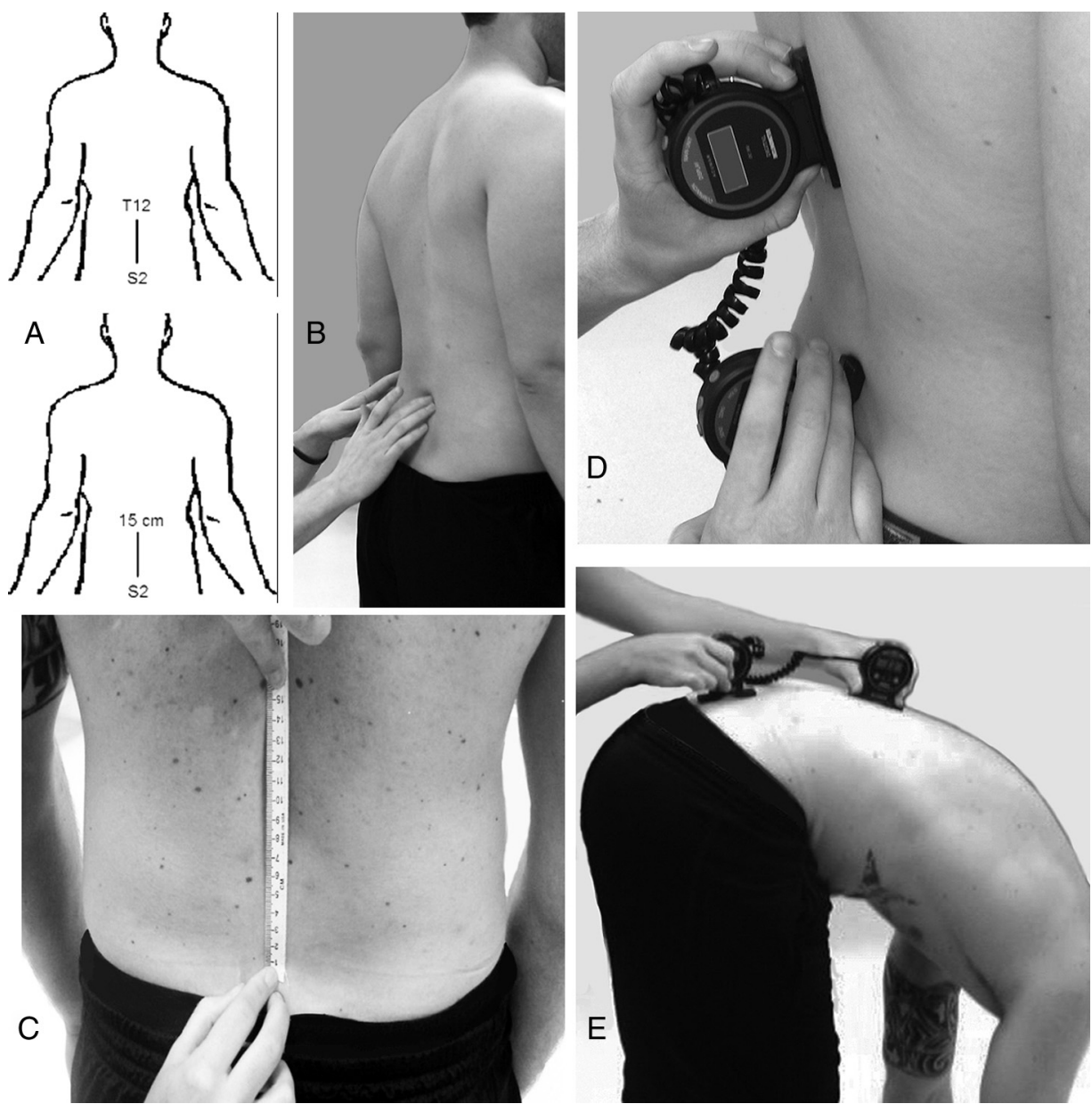

Figure 1 - Measurement techniques. (A) Landmarks for placement of the HDDI; T12 palpation technique versus 15-cm technique. (B) Process of locating T12 by palpation; shows palpation of the upper lumbar region. (C) Approximation of T12 by the 15-cm technique. (D) Placement of HDDI device with subject standing upright in self-selected comfortable position. (E) Measurement of lumbar flexion active range of motion using HDDI device. HDDI indicates handheld digital dual inclinometry.

Table 1 Descriptive Statistics $(n=69)$

\begin{tabular}{lcccc}
\hline & Mean & Minimum & Maximum & SD \\
\hline Age, y & 23.8 & 21 & 34 & 2.38 \\
Body height, cm & 172.13 & 156 & 193.5 & 9.13 \\
Body weight, kg & 71.20 & 48.07 & 108.40 & 14.52 \\
Body mass index & 23.93 & 17.34 & 32.01 & 3.53 \\
\hline
\end{tabular}

easier and more convenient of the 2 techniques and is potentially just as, if not more, reliable within a single examiner.

A study comparing the HDI $15-\mathrm{cm}$ technique to measuring lumbar movement using sagittal plane spine radiographs showed strong test-retest reliability for HDI of lumbar flexion $(\mathrm{ICC}=.76)$, in comparison to the reliability of the radiograph-based measurement (ICC $=.78$ ), and a correlation between the 2 measurements of $r=.67 .{ }^{10} \mathrm{~A}$ study comparing the HDI T12 palpation technique with and without fluoroscopy assistance to find the $\mathrm{T} 12$ spinous process found a close correlation $(r=.95)$ between the lumbar-flexion measures. ${ }^{11}$ MacDermid et $\mathrm{al}^{8}$ demonstrated more sources of error potentially impacting the T12 palpation technique, yet determined that both the $15 \mathrm{~cm}$ and the T12 palpation techniques each had strong reproducibility for measurement of lumbar flexion AROM. MacDermid et $\mathrm{al}^{8}$ did not present any statistical analysis for correlation or clinical comparison between the $2 \mathrm{HDI}$ measurement techniques.

Both the statistical significance and clinical importance of the study findings were considered. A statistically significant association was found between the 2 HDDI techniques $(r=.80, P<.001)$, which can be inferred as a strong correlation. To reflect the comparison in terms of clinical relevance, the degree of variability in scores between the 2 methods was examined using the $95 \%$ LOA. Based on the $95 \%$ LOA, a clinician can expect the difference in measuring lumbar flexion AROM between the 2 methods to vary between $-13.1^{\circ}$ and $10.7^{\circ}$.

This study was conducted in a controlled environment with asymptomatic subjects; thus, caution should be used in applying the results to a symptomatic population. Furthermore, the results should not be applied to younger populations who are not skeletally mature. Future research should compare the 2 HDDI methods within larger subset groups based on body height and body mass index. Finally, an investigation comparing the 2 HDDI methods within the larger context of lumbar extension, as well as lumbar lateral flexion and rotation motions, is needed. In conclusion, the findings suggest that, for an experienced examiner using 


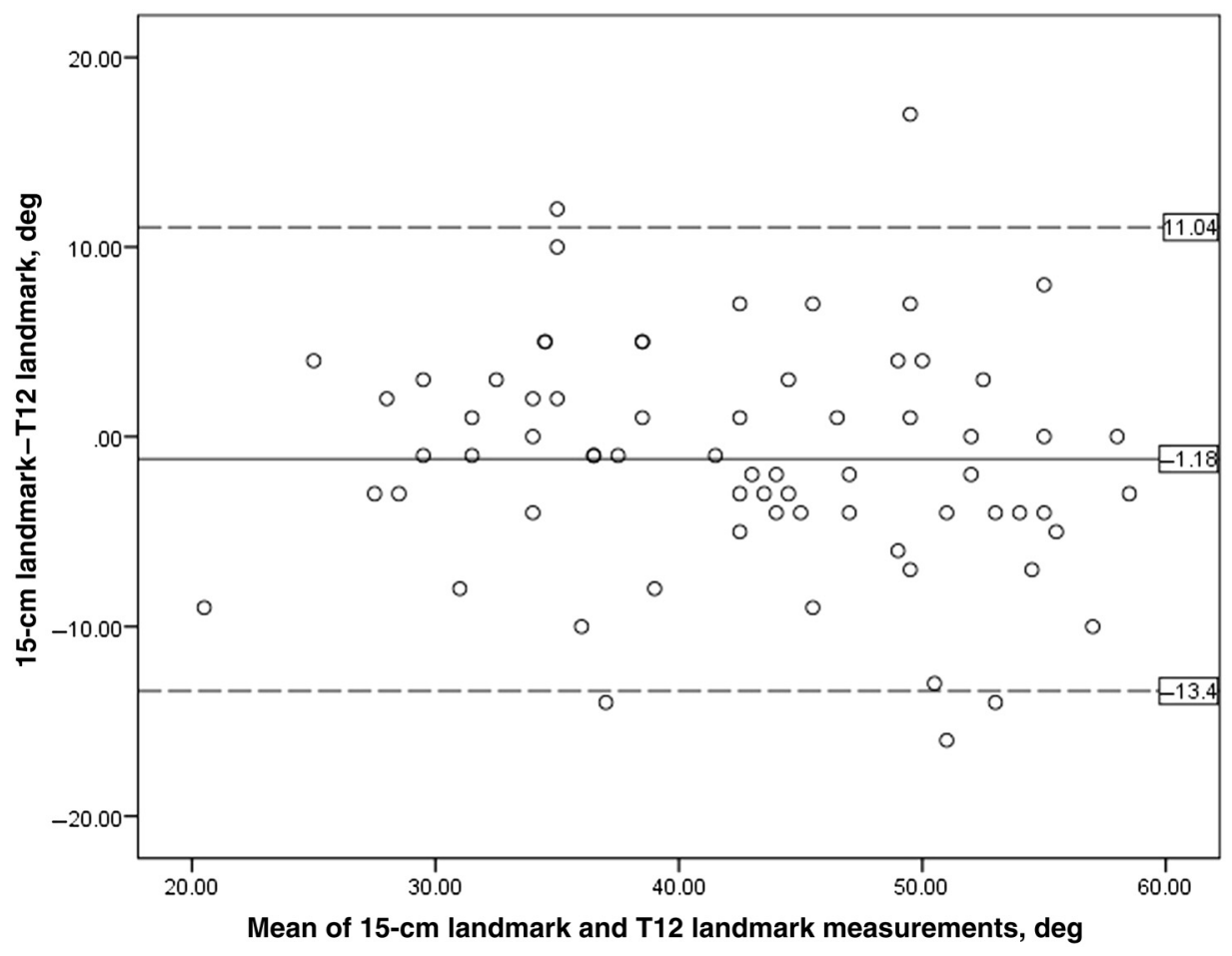

Figure 2 - Bland-Altman plot with $95 \%$ limits of agreement. The solid line is the mean difference between the 2 techniques. The dotted lines represent the $95 \%$ limits of agreement (+1.96 SD).

a standardized, reliable method of HDDI to measure lumbar flexion AROM, results are comparable in magnitude when using either of the 2 HDDI techniques described on healthy adults.

\section{References}

1. Sullivan MS, Shoaf LD, Riddle L. The relationship of lumbar flexion to disability in patients with low back pain. Phys Ther. 2000; 80(3):240-250. PubMed ID: 10696151 doi:10.1093/ptj/80.3.240

2. Cocchiarela L, Anderson GBJ. Guides to the Evaluation of Permanent Impairment. 5th ed. Chicago, IL: American Medical Association; 2000.

3. Reese N, Bandy W. Joint Range of Motion and Muscle Length Testing. 3rd ed. St Louis, MO: Elesevier Inc; 2007.

4. Madson T, Youdas J, Suman V. Reproducibility of lumbar spine range of motion measurements using the back range of motion device. J Orthop Sports Phys Ther. 1999:29(8):470-477. PubMed ID: 10444737 doi:10.2519/jospt.1999.29.8.470

5. Williams R, Binkley J, Bloch R, Goldsmith $\mathrm{CH}$, Minuk T. Reliability of the modified - modified Schober and double inclinometer methods for measuring lumbar flexion and extension. Phys Ther. 1993;73(1): 26-37. PubMed ID: 8417457 doi:10.1093/ptj/73.1.26
6. Tousignant M, Poulin L, Marchand S, Viau A, Place C. The modifiedmodified Schober test for range of motion assessment of lumbar flexion in patients with low back pain: a study of criterion validity, intra- and inter-rater reliability and minimum metrically detectable change. Disabil Rehabil. 2005;27(10):553-559. PubMed ID: 16019864 doi:10.1080/09638280400018411

7. Lafayette Instrument. Acumar Digital Inclinometer User's Manual. Lafayette, IN: Lafayette Instrument Co; 2013.

8. MacDermid JC, Arumugam V, Vincent JI, Payne KL, So AK. Reliability of three landmarking methods for dual inclinometry measurements of lumbar flexion and extension. BMC Musculoskelet Disord. 2015;16:121-126. PubMed ID: 25989834 doi:10.1186/ s12891-015-0578-2

9. Portney LG, Watkins MW. Foundations of Clinical Research. 3rd ed. Philadelphia, PA: FA Davis Co; 2015.

10. Williams RM, Goldsmith $\mathrm{CH}$, Minuk T. Validity of the double inclinometer method for measuring lumbar flexion. Physiother Can. 1998;50:147-152.

11. Saur PM, Ensink FM, Frese K, Seeger D, Hildebrandt J. Lumbar range of motion: reliability and validity of the inclinometer technique in the clinical measurement of trunk flexibility. Spine. 1996;21(11): 1332-1338. PubMed ID: 8725925 doi:10.1097/00007632-19960 6010-00011 